\title{
Understanding the effect of cube size on the near wake characteristics in a turbulent boundary layer
}

\author{
Siddhesh Shinde, Eric Johnsen ${ }^{\dagger}$ and Kevin Maki ${ }^{\ddagger}$ \\ University of Michigan, Ann Arbor, MI, 48109, USA
}

\begin{abstract}
Wall-resolved large-eddy simulation of flow over a wall-mounted cube-shaped obstacle placed in a spatially evolving boundary layer is performed. The Reynolds number of the flow based on the mean velocity and the inlet boundary layer thickness is 19,600. Our interests lie in understanding how variations in the cube height $h$ modify the flow dynamics for situation where the cube is within the boundary layer. For this purpose, we conduct simulations with height to boundary layer thickness ratios of $h / \delta_{o}=0.2, \mathbf{0 . 6}$ and 1.0, where $\delta_{o}$ is the boundary layer thickness at the cube location. The size of the vortices formed around the cube, the Reynolds stresses and the turbulent kinetic energy in the boundary layer all depend on the cube height relative to the boundary layer thickness. The drag coefficient of the cube is found to increase with increasing $h / \delta_{o}$.
\end{abstract}

\section{Introduction}

Flow around bluff bodies is a common occurrence in engineering applications and plays a vital role in the engineering design process, for instance, estimating the wind load over a building or predicting the aerodynamic drag acting on a vehicle. An example of a simple flow configuration is that of a cube placed in fully developed channel flow; this problem has been used as a benchmark case by researchers to validate their numerical methods and turbulence models. ${ }^{1-6}$ Unsteady incompressible flow predictions of wall-mounted cube placed in a spatially evolving turbulent boundary layer is a more challenging problem to simulate accurately due to the complex vortical structures around the cube. Experimental and numerical studies have revealed that a horse-shoe vortex is formed in front of the cube, a hair-pin vortex on the top of the cube, tornado-like vortices on the sides and vortex shedding in the wake. These features are illustrated qualitatively in figure 1, taken from one of our representative simulations.

Martinuzzi et al. ${ }^{7}$ performed an extensive experimental study of flow over surface-mounted prismatic obstacles in a fully developed channel flow. Their experiments were performed at Reynolds numbers ranging from 80,000 to 115,000 based on the channel height. They considered prismatic obstacles in the shape of a rectangular block with width to height $(W / H)$ ratio ranging from 1 to 24 and documented the reattachment length and separation length for different ratios. They concluded that for $W / H>6$, the flow is nominally 2 dimensional in the middle of the wake. In a experimental study by Hussein and Martinuzzi ${ }^{8}$ the evolution of the spatial dissipation rate from the near field recirculation zone to the asymptotic wake is examined.

Numerical studies of flow over a wall-mounted cube placed in a turbulent channel have been performed using Reynolds Averaged Navier Stokes (RANS) simulations of this flow configuration, including Rodi et al. ${ }^{4}$ and Iaccarino et al. ${ }^{5}$ These studies suggest that steady RANS simulations cannot predict this flow accurately as unsteady effects due to vortex shedding are neglected. Shah et al. ${ }^{2,9}$ and Krajnovic et al. ${ }^{1,3}$ performed Large-Eddy Simulation (LES) of this problem at a Reynolds number of 40,000 based on the mean velocity and cube height. They compared their results with those of Martinuzzi et al. ${ }^{7}$ with good agreement. For this problem, the one-equation LES model and its variants were reported to perform better than the Smagorinsky type models due their ability to capture back-scatter.

\footnotetext{
${ }^{*}$ Graduate Student, Department of Mechanical Engineering, 1231 Beal Ave., 2043 Walter E. Lay Automotive Laboratory, Ann Arbor, MI 48109-2133, AIAA member.

${ }^{\dagger}$ Assistant Professor, Department of Mechanical Engineering, 1231 Beal Ave., 2043 Walter E. Lay Automotive Laboratory, Ann Arbor, MI 48109-2133, AIAA senior member.

${ }^{\ddagger}$ Assistant Professor, Naval Architecture and Marine Engineering, 210 NAME Building, 2600 Draper Drive, Ann Arbor, MI 48109-2145.
} 


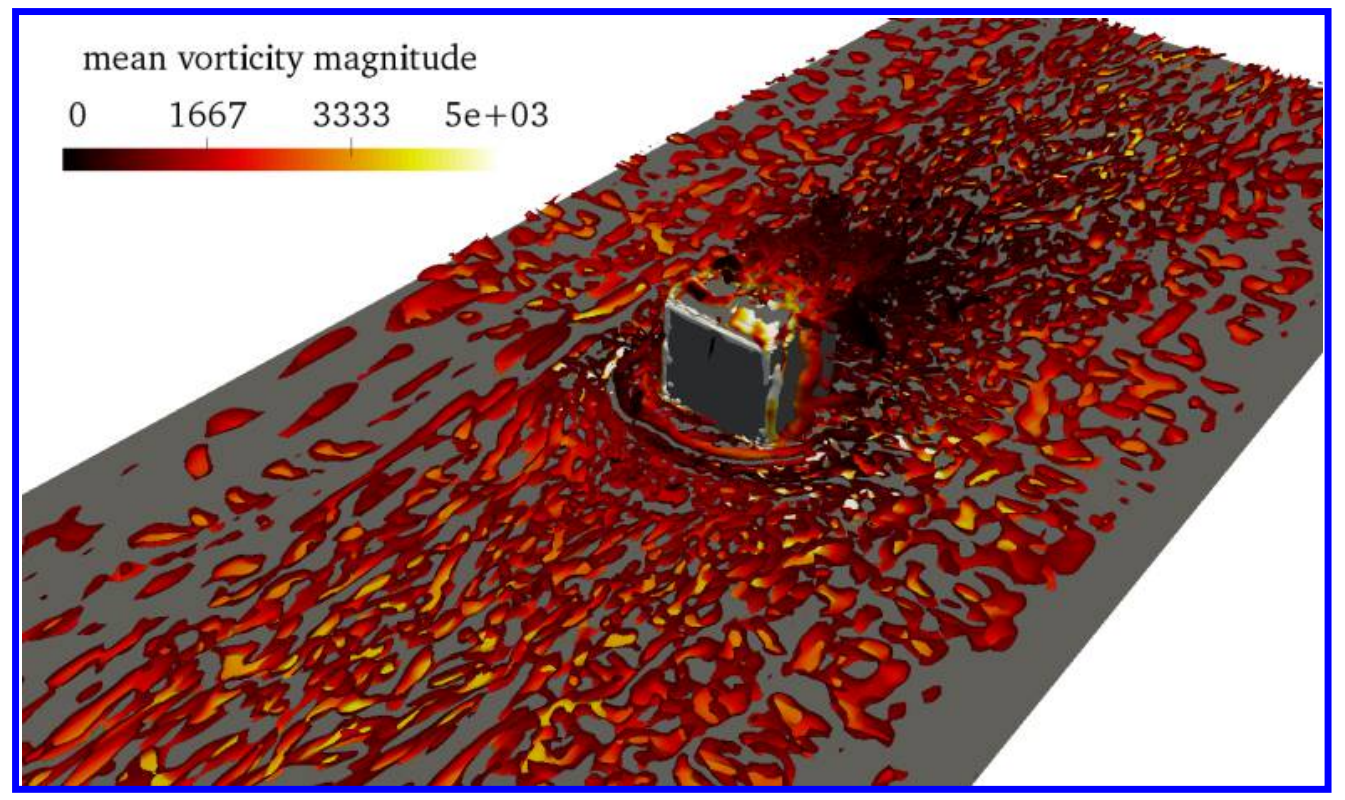

Figure 1. Second invariant of the instantaneous velocity field for flow around a wall-mounted cube in a spatially evolving turbulent boundary layer

More recently, Liakos et al. ${ }^{10}$ performed a Direct Numerical Simulation (DNS) of steady-state laminar flow over a cube at Reynolds numbers ranging from 1-2000 based on the cube height. The study investigates the onset of dominant flow structures and instability in this flow configuration. It was found that the horse-shoe vortex begins to appear at a Reynolds number of 1267 and the onset of unsteadiness is seen at $R e=2000$. In another DNS study by Yakhot et al. ${ }^{11,12}$ the inflow was fully turbulent with a Reynolds number of 1870 based on the cube height. In this study, the unsteadiness in this flow is reported to be caused by the viscous-inviscid interaction between the horse-shoe vortex and a narrow band of positive vorticity in front of the cube.

The literature demonstrates that there is a good understanding of the complex flow structures formed around the cube like the horse-vortex, hair-pin vortices and vortex shedding for a range of Reynolds numbers. However, most of these studies are performed in a fully developed channel flow with a cube height approximately half the channel height. We are interested in a situation where an obstacle is being placed in a spatially evolving turbulent boundary layer. There have been some efforts in the past by researchers in the atmospheric boundary layer community to perform numerical studies of cubic obstacles placed in a turbulent boundary layer (Yazid et al. ${ }^{6}$ Lim et al. ${ }^{13}$ ). However, these efforts either use the RANS approach or wall-modeled LES with a rough wall model used to replicate the bottom-wall. Flow around a wall-mounted cube in a zero pressure gradient flat plate boundary layer (ZPGFPBL) has not garnered significant attention, particularly using wall-resolved LES. This lacuna of knowledge motivates the present study. The goal of our work is to understand the effect of varying the cube height $(h)$ to boundary layer thickness $\left(\delta_{o}\right)$ ratio on the turbulent wake characteristics. To adequately represent the physics, we used wall-resolved LES.

The article is organized as follows. We first describe our numerical approach in Section II. We then present validation results for a cube in a fully developed turbulent channel flow and for a spatially evolving zero-pressure gradient flat plate turbulent boundary layer in Section III. Next, we investigate the flow dynamics of a wall-mounted cube in a turbulent boundary layer in Section IV. We outline our conclusions and list future research directions in Section V.

\section{Numerical approach}

The incompressible Navier-Stokes equations are solved using the one equation eddy viscosity LES model in OpenFOAM. A second order central finite volume scheme is used in space and the solution is advanced in time using a second order backward implicit time marching scheme. We validate the discretization schemes and LES model in OpenFOAM by simulating flow over a wall-mounted cube in a fully developed channel and 


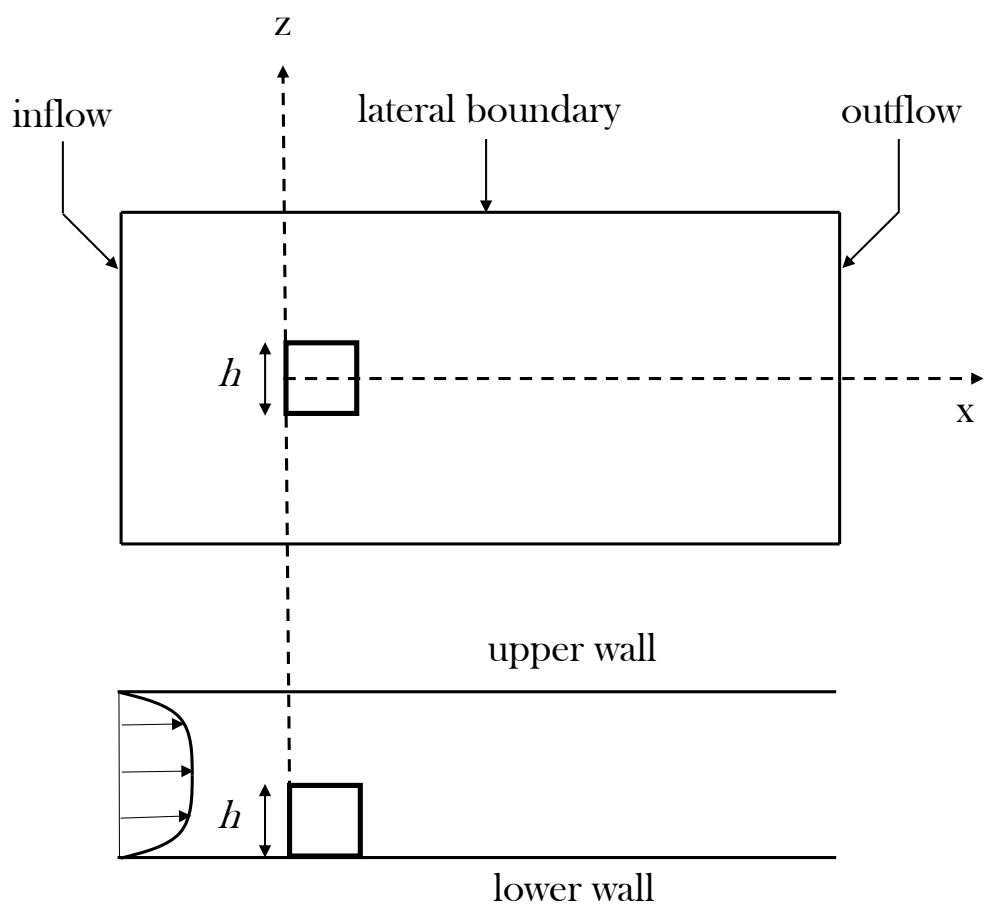

Figure 2. Schematic of the case setup ${ }^{1,8}$ for flow over a wall-mounted cube in a channel.

comparing our results with previous numerical ${ }^{1}$ and experimental ${ }^{8}$ studies. We validate our inflow generator by simulating a spatially evolving turbulent boundary layer at the desired Reynolds number of 19,600 and comparing the turbulent statistics with the work done by $\mathrm{Wu}$ et al. ${ }^{14}$

\section{Validation}

Given the lack of relevant experimental data for the problem of interest, we conduct our validation studies for two related problems: fully developed turbulent flow around a wall-mounted cube in a channel, and spatial evolution of a zero-pressure gradient flat plate turbulent boundary layer.

\section{III.A. Fully developed turbulent flow over a wall-mounted cube in a channel}

Experimental $^{8}$ and numerical ${ }^{1,9}$ studies have been performed for fully developed flow over a wall-mounted cube placed in a channel. The reason we chose to compare our results to those of Krajnovic et al.'s ${ }^{1}$ numerical study as our validation case is that they used similar LES models and discretization schemes as ours. For these reasons this test case would provide us with valuable information about the accuracy of our flow solvers and the LES model.

Full details about the geometry and the numerical setup can be found in Krajnovic et al. ${ }^{1}$ A precursor channel flow simulation at $R e_{b}=40,000$ (based on the mean velocity and half channel height) was performed to obtain a single inlet velocity profile to be used as the inlet boundary condition. We used the finest mesh resolution as used by Krajnovic et al. such that, $\Delta x^{+}=28.5, \Delta y^{+}=4.6$ and $\Delta z^{+}=23.7$ in the vicinity of the cube.

The separation $\left(X_{F}\right)$ and reattachment $\left(X_{R}\right)$ lengths for the simulation as presented in table 1 were calculated from the skin-friction coefficient $C_{f}=\frac{\tau_{w}}{1 / 2 \rho U_{b}^{2}}$ in the center-plane along the bottomwall of the domain. We obtain a $10 \%$ error in $X_{F}$ and a $13 \%$ error in $X_{R}$ relative to the experiments, by contrast to 9.1\% error in $X_{F}$ and $9.7 \%$ error in the LES by Krajnovic et al. ${ }^{1}$ This discrepancy is partly due to the coarse grid near the separation and reattachment point, as local mesh resolution plays a larger role in predicting the correct reattachment length than in predicting the flow over the top of the cube. ${ }^{1}$

In figure 3 we show the $x$ - and $y$-components of the mean velocity and the $x x$ - and $x y$-components 

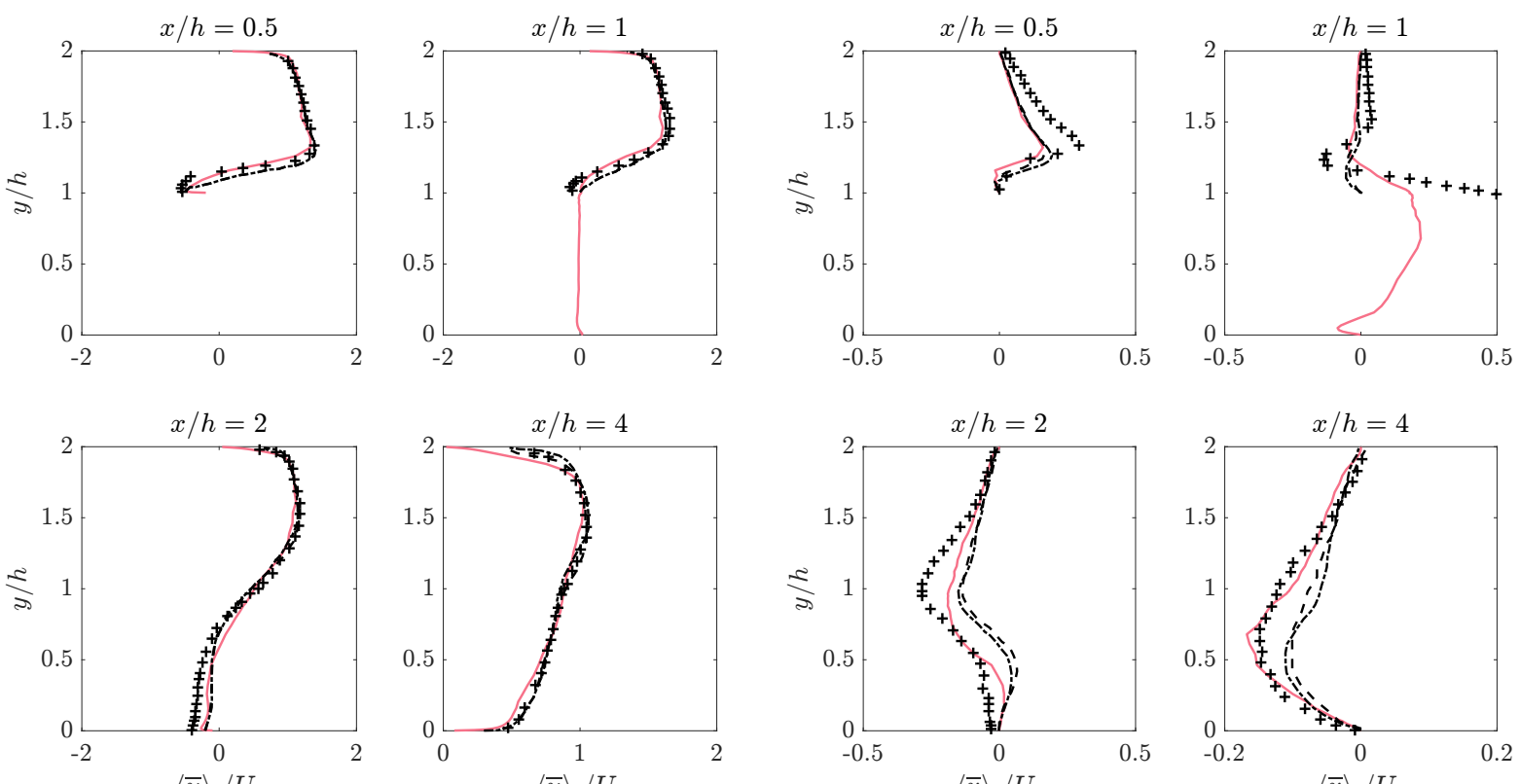

(a) $x$-component of mean velocity

(b) $y$-component of mean velocity
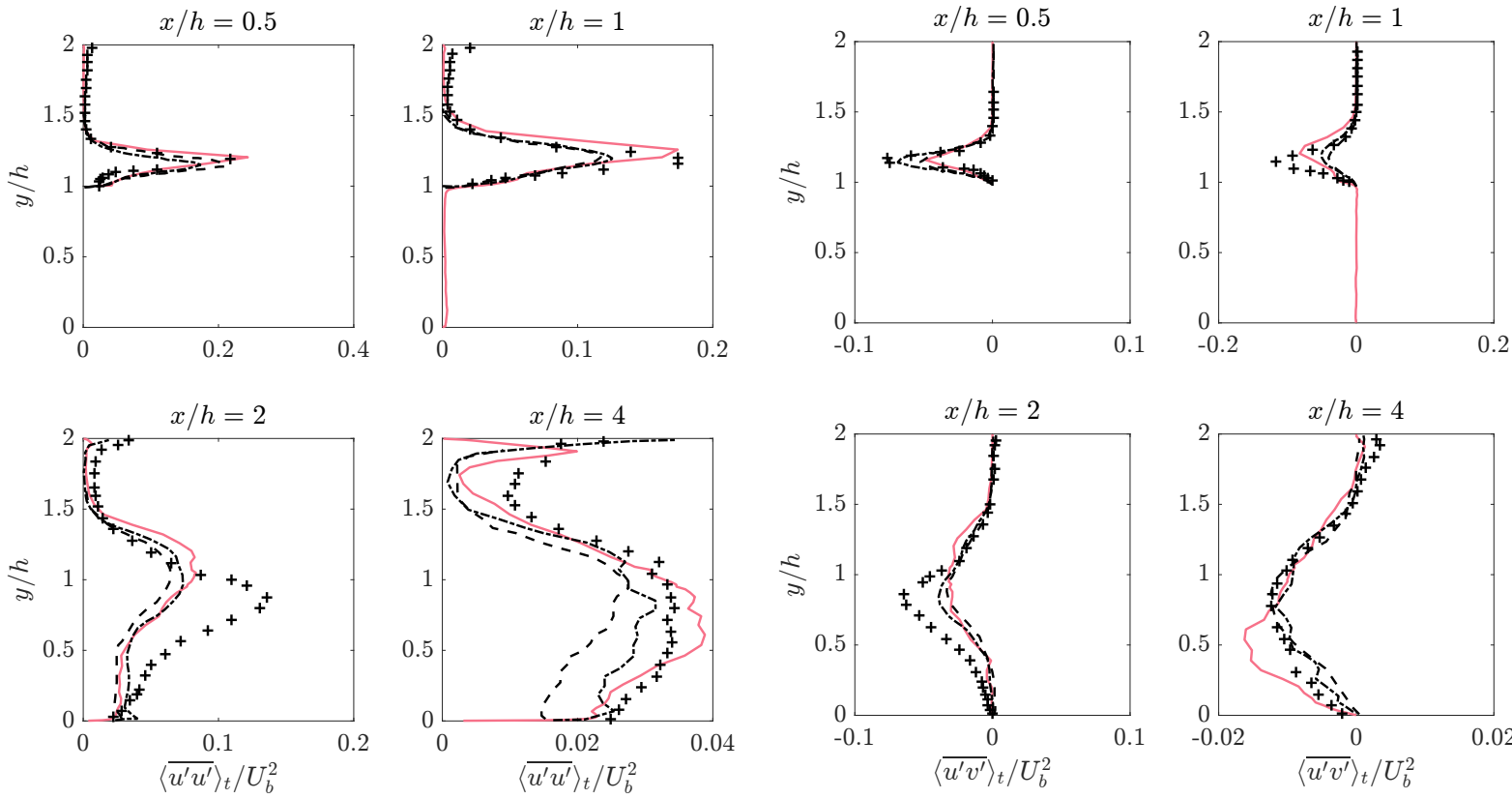

(c) $x x$-component of Reynolds stress
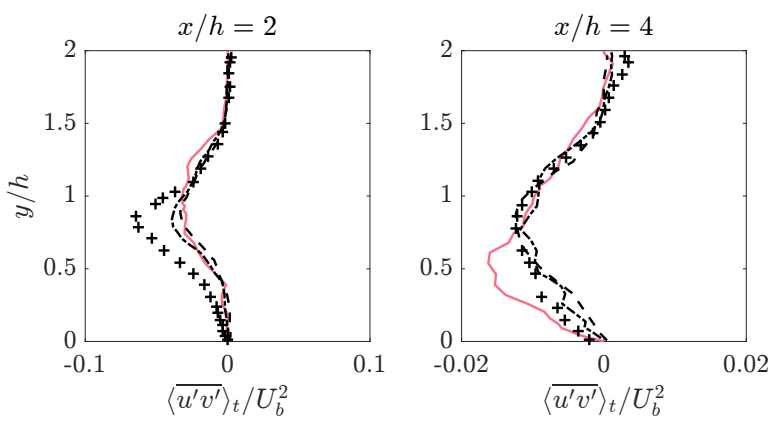

(d) $x y$-component of Reynolds stress

Figure 3. Mean velocity components and Reynolds stresses in the center plane for fully developed turbulent flow around a wall-mounted cube in a channel ++ : experimental result ${ }^{8}$ dashed line: one equation model $;^{1}$ dash-dotted line: localized dynamic one equation model $;^{1}{ }_{-}$: our results with one equation model.

of the Reynolds stress. In general, the agreement of our results with the experiments is at least as good, if not better, than Krajnovic et al., ${ }^{1}$ except for the $x y$-component of the Reynolds stress tensor. We expect that the agreement with experiments would be even better with finer resolution simulations.

\section{III.B. Zero pressure gradient flat plate turbulent boundary layer}

To verify the accuracy of the inflow conditions at desired Reynolds number we simulate a zero pressure gradient flat plate turbulent boundary layer (ZPGFPBL). The objective of this simulation was two-fold: to test the accuracy of the inflow conditions replicating a ZPGFPBL at a Reynolds number (based of bulk 


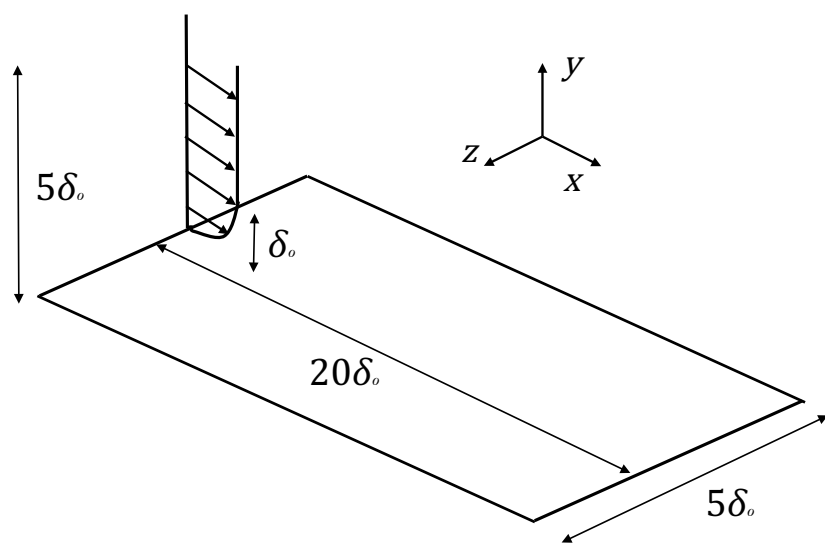

Figure 4. Schematic of the case setup for zero pressure gradient flat plate boundary layer simulations

velocity $U_{b}$ and boundary layer thickness at the inlet $\delta_{o}$ ) of 19,600, and to determine the properties of the boundary layer as a function of streamwise coordinate to place the cube at the appropriate location in our subsequent simulations.

Based on the friction velocity $\left(u_{\tau}\right)$ and $\delta_{o}$, the Reynolds number is 800 . As seen in figure 4 the length of the domain is $20 \delta_{o}$, the height is $5 \delta_{o}$ and the width is $5 \delta_{o}$. The mesh is uniform in the streamwise $(x)$ and the spanwise $(z)$ directions whereas, in the wall-normal $(y)$ direction it is non-uniform. In wall-units the grid spacing is, $\Delta x^{+}=23, \Delta y_{\text {wall }}^{+}=2$ and $\Delta z^{+}=23$. A no-slip boundary conditions is prescribed on the bottom-wall, slip boundary condition on the top-wall. The domain is periodic in the spanwise direction. A zero-gradient boundary condition is prescribed at the outlet for velocity and the inlet boundary condition is a time-varying turbulent inflow condition as describe by Shinde et al. ${ }^{15}$ The simulation is run for $1200 \delta_{o} / U_{b}$ time units and the turbulent statistics are averaged over $400 \delta_{o} / U_{b}$ units.

In figure 5 , we show the boundary layer thickness $\left(\delta / \delta_{o}\right)$ and momentum thickness Reynolds number $\left(R e_{\theta}=U_{\infty} \theta / \nu\right)$ as a function of the streamwise coordinate $(x)$. From Direct Numerical Simulation (DNS) of a spatially evolving turbulent boundary layer done in prior studies ${ }^{14,16}$ we expect $\delta / \delta_{o}$ and $R e_{\theta}$ to grow in a linear manner with $x$ after a brief transition length in the beginning of the domain, which is the behavior observed in the figures $5(\mathrm{a})$ and $5(\mathrm{~b})$. In our subsequent simulations, we place the cube at $x / \delta_{o}=12$, since $\delta / \delta_{o}=1$

We further compare the Reynolds stresses at different streamwise locations in figure 6 with DNS results from Wu et al. ${ }^{14}$ The DNS results are obtained at a momentum thickness Reynolds number $\left(R e_{\theta}=U_{\infty} \theta / \nu\right)$ of 900 whereas in our simulation $R e_{\theta}$ varies from 2100 to 2600 . As described by Wu et al. and De Graaff et al. ${ }^{17}$ the Reynolds stresses do not exhibit a universal scaling in inner coordinates especially in the logarithmic region and hence, we do not expect our simulation results to match exactly. However, after an initial transition length of approximately $10 \delta_{o}$ there is negligible difference in streamwise $\left(\overline{u^{\prime 2+}}\right)$ Reynolds stress and the wall-normal $\left(\overline{v^{\prime 2+}}\right)$ Reynolds stress. The near-wall peak $\overline{u^{\prime 2+}}$ value matches well in location with the DNS data, as expected.

From the behavior of mean and turbulence quantities in figures 5 and 6 we are confident that we can

\begin{tabular}{lcccc}
\hline \hline Contribution & LES Model & Grid & $X_{F} / h$ & $X_{R} / h$ \\
\hline Martinuzzi and Tropea $^{8}$ & Experiment & - & 1.04 & 1.61 \\
Krajnovic et al. $^{1}$ & one equation & $210 \times 66 \times 114$ & 1.10 & 1.45 \\
Krajnovic et al. $^{1}$ & localized dynamic one eqn. & $210 \times 66 \times 114$ & 1.13 & 1.44 \\
Present simulations & one equation & $210 \times 66 \times 114$ & 0.936 & 1.82 \\
\hline \hline
\end{tabular}

${ }^{*} X_{F}$ : separation length in front of the cube, $X_{R}$ : reattachment length behind the cube

Table 1. Separation and reattachment lengths normalized by the cube height $(h)$ 


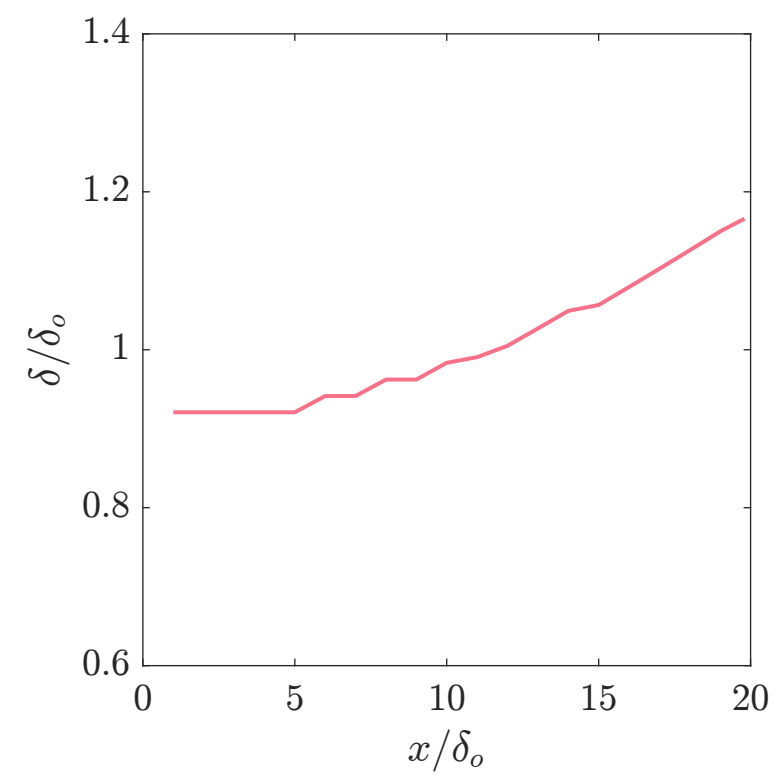

(a) Boundary layer thickness as a function of the streamwise (b) Reynolds number based on the momentum thickness as a coordinate

Figure 5. Integral quantities averaged in time and the spanwise direction, normalized by boundary layer thickness at the inlet $\left(\delta_{o}\right)$

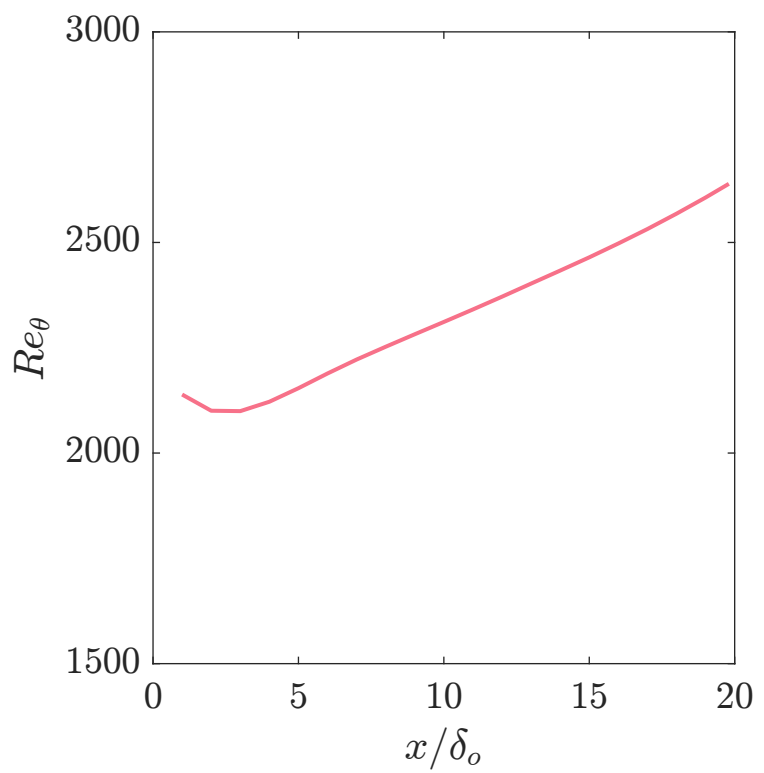
function of the streamwise coordinate (a) $x x$-component

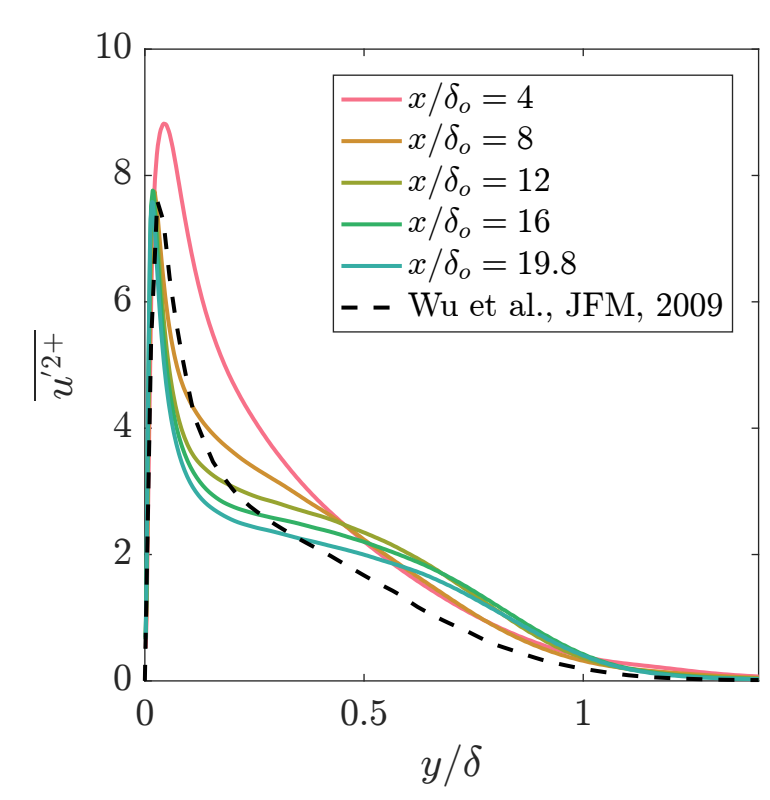

Figure 6. Reynolds stress at different streamwise locations

simulate a spatially evolving turbulent boundary layer with reasonable accuracy and that the boundary layer is turbulent at a streamwise location, $x / \delta_{o}=12$ where the boundary layer thickness is $\delta_{o}$. 


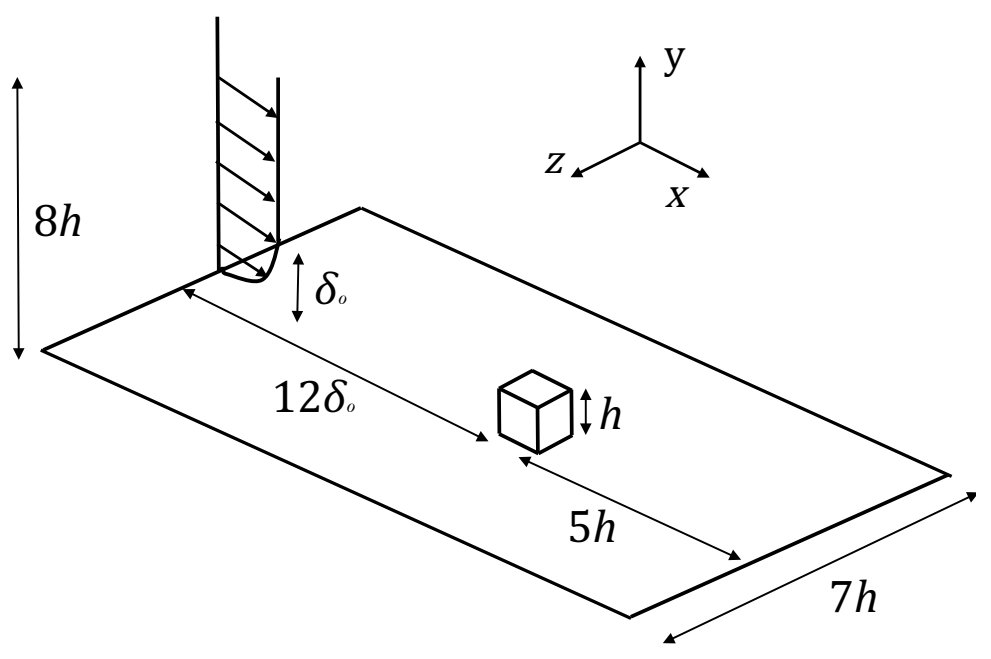

Figure 7. Schematic of the case setup for spatially evolving turbulent boundary layer flow around a wall-mounted cube.

\section{Flow around a cube in a spatially evolving turbulent boundary layer}

\section{IV.A. Case setup}

As seen in figure 7 the cube is placed in the plane of symmetry of the domain. The cube height is $h$ and the boundary layer thickness at the inlet of the domain is $\delta_{o}$. Three different cube height to boundary layer thickness ratios $\left(h / \delta_{o}\right)$ are used: $0.2,0.6$ and 1. The length of the domain in front of the cube is $12 \delta_{o}$ and the length of the domain behind the cube is $5 h$. A length of $12 \delta_{o}$ was chosen in front of the cube because our ZPGFPBL simulations predict that at this distance from the inlet the boundary layer is turbulent and the boundary layer thickness is $\delta_{o}$. The choice of spanwise width of $7 h$ is based on our knowledge from prior studies. ${ }^{1}$ This width is considered to be sufficient to prevent sidewalls effects from becoming significant. The domain height is $8 h$. The Reynolds number $\left(R e_{b}\right)$ based on the bulk velocity $\left(U_{b}\right)$ and boundary layer thickness $\left(\delta_{o}\right)$ at the inlet is 19,600 . The Reynolds number $\left(R e_{\tau}\right)$ based on the friction velocity $\left(u_{\tau}\right)$ and the boundary layer thickness $\left(\delta_{o}\right)$ at the inlet is 800 . The simulation is carried out for a total time period of $300 \delta_{o} / U_{m}$ with variable time-step and a maximum Courant number of 1 . The turbulent statistics are averaged over a time period of $100 \delta_{o} / U_{m}$ to avoid contamination of the results by the initial transient.

The boundary conditions used in the simulation are no-slip on the bottom-wall and the surface of the cube. Slip-wall boundary condition was used on the side walls and the top wall. A zero-gradient boundary condition is used on the outlet. A time varying turbulent inflow boundary condition as described by Shinde et al. ${ }^{15}$ is used at the inlet in order to obtain a spatially evolving turbulent boundary layer. The mesh is non-uniform with smallest cells in the vicinity of the cube and largest cells towards the periphery of the domain. The grid spacing of cells next to the cube are given in table 3. A hyperbolic tangent stretching is used for the cells in the streamwise and spanwise direction. In the wall normal direction the grid spacing on the bottomwall and the top surface of the cube is as described in table 3. This fine resolution is required to resolve the fine scales inside the turbulent boundary layer and those near the top surface of the cube. The cells are stretched using a hyperbolic tangent spacing from the top surface of the cube to the top of the domain. For each $h / \delta_{o}$ ratio three meshes with different levels of refinement are used.

\section{IV.B. Results and discussion}

We compared the streamlines in the plane of symmetry of the domain and, in the plane at $y=0.1 h$ from the bottom-wall for different cube heights in figure 8. We observe the vortices reported in previous studies: the horseshoe vortex formed in front of the cube, the hair-pin vortex formed on the top the cube and counter rotating vortex pair formed behind the cube. The arch-type vortex formed behind the cube as seen in the left column in figure 8 is larger for $h / \delta_{o}=0.2$ than those observed in the other cube heights. We believe the reason for this larger vortex is the location inside the boundary layer: the vortex for $h / \delta_{o}=0.2$ is closer to the wall, where the velocity magnitudes are smaller as compared to the vortices for $h / \delta_{o}=0.6$ and 


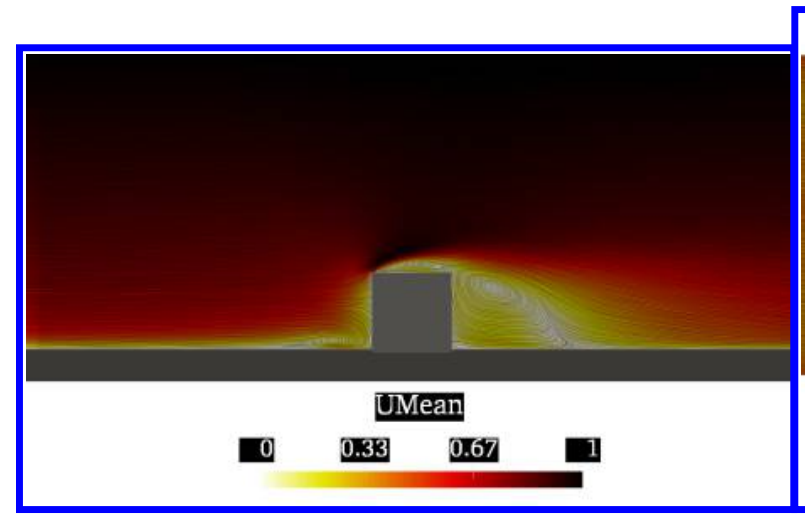

(a) $h / \delta_{o}=0.2$, centerplane

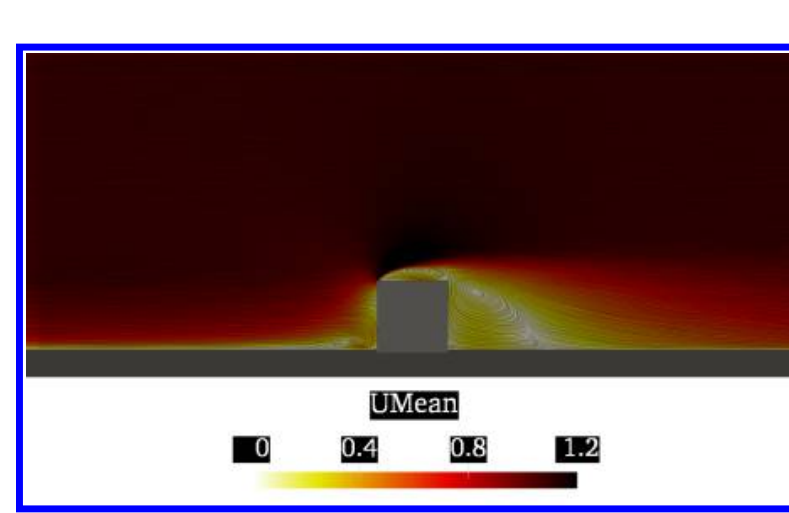

(c) $h / \delta_{o}=0.6$, centerplane

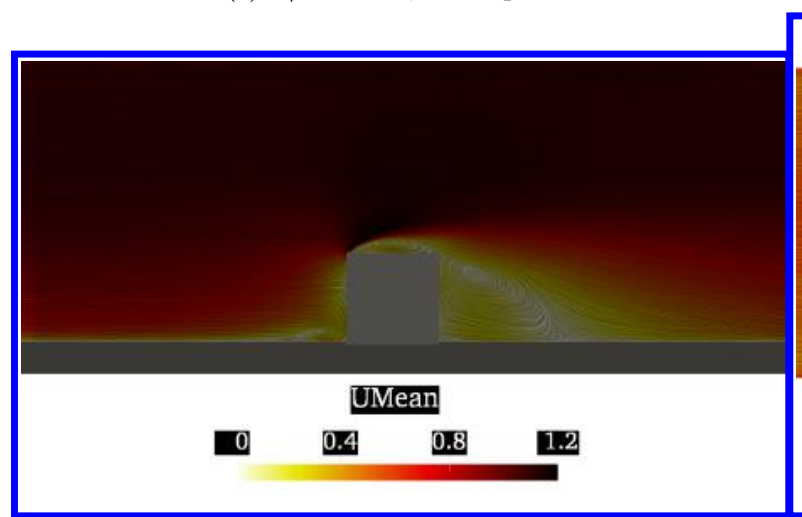

(e) $h / \delta_{o}=1.0$, centerplane

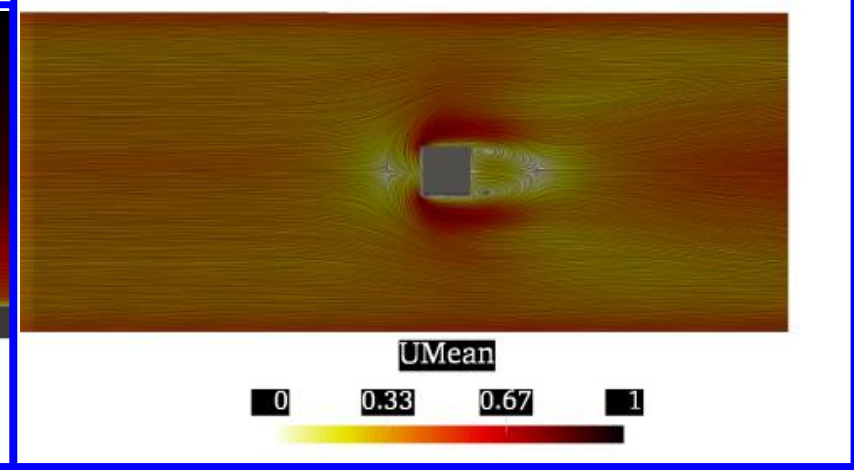

(b) $h / \delta_{o}=0.2$, at $y / h=0.1$

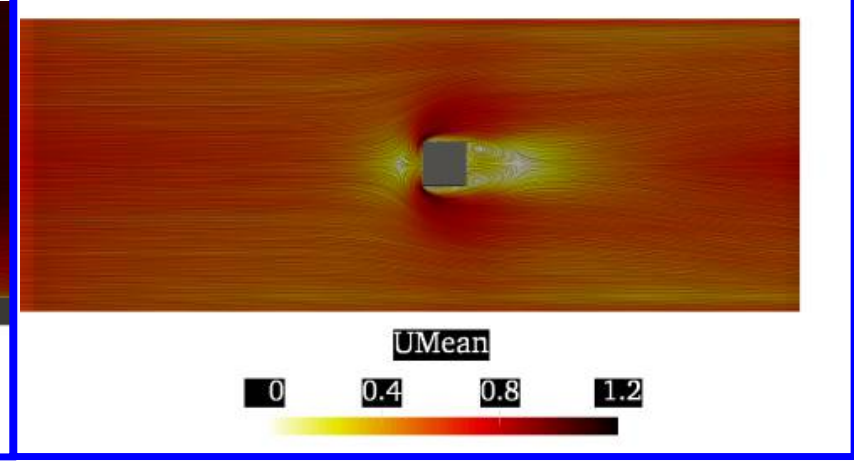

(d) $h / \delta_{o}=0.6$, at $y / h=0.1$

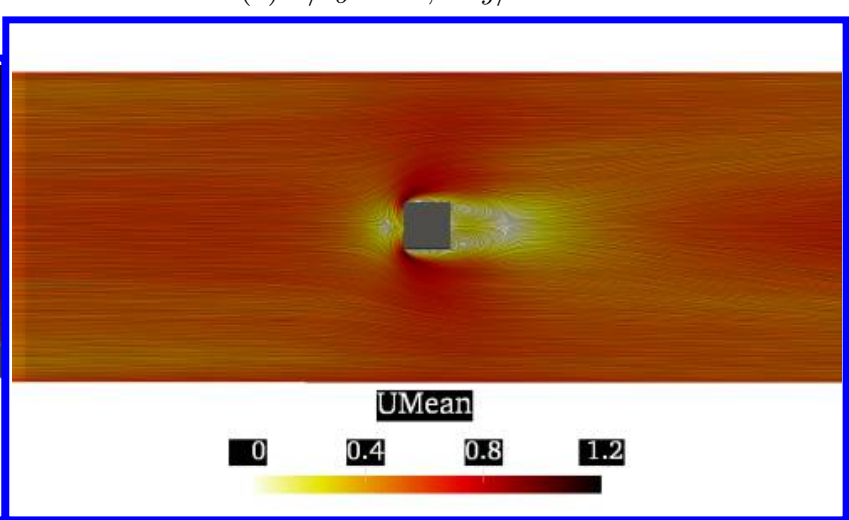

(f) $h / \delta_{o}=1.0$, at $y / h=0.1$

Figure 8. Streamlines colored by speed for spatially evolving turbulent boundary layer flow over a wall-mounted cube.

1.0. There exist 2 saddle points in the flow, first, where the flow separates in front of the cube and second, where the flow reattaches behind the cube. The streamlines diverge from these points in the flow field. The horse-shoe vortex extends to a distance of $\sim 1.5 h$ on the sides of the cube in all three cases. Yakhot et al. ${ }^{11}$ observe similar width of the horse-shoe in DNS of flow over a wall-mounted cube in a channel.

By sampling the $x$-component of mean velocity along the bottom-wall, the skin friction coefficient $\left(C_{f}\right)$ is plotted as a function of the stream-wise coordinate in figure 9 . As the flow gets separated in front of the cube, $C_{f}$ becomes negative; this length is defined at the separation length $\left(X_{F}\right)$. The flow reattaches after the arch-type vortex behind the cube at the point where $C_{f}$ becomes positive again; this length is called the reattachment length $\left(X_{R}\right)$. The values of $X_{F}$ and $X_{R}$ for different $h / \delta_{o}$ ratios are presented in table 2 . The non-dimensional values of $X_{F}$ and $X_{R}$ are almost identical for different $h / \delta_{o}$ ratios. This suggests their independence on the $h / \delta_{o}$ ratio.

The drag coefficient $\left(C_{d}\right)$ on the other hand increases with increase in cube height. This is expected for 


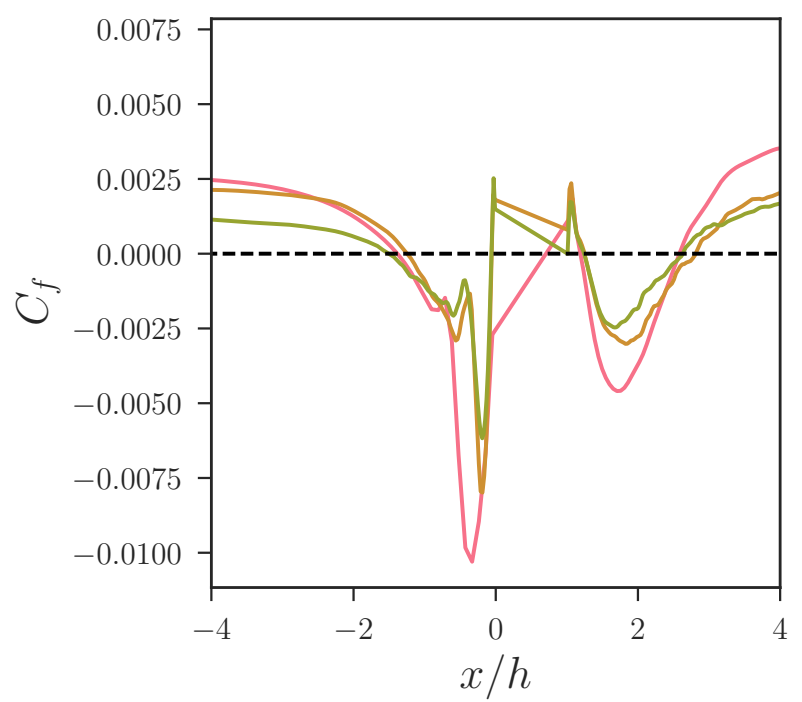

Figure 9. Skin friction coefficient $\left(C_{f}\right)$ along the bottomwall for a spatially evolving turbulent boundary layer flow around a wall-mounted cube. $-h / \delta_{o}=0.2,-h / \delta_{o}=0.6$ and $-h / \delta_{o}=1.0$.

2 reasons: a larger cube presents a larger area normal to the flow, thus causing a greater pressure force on the front face, and a larger cube also has a larger surface area, thus increasing the total viscous force acting on the cube. The values of $C_{d}$ for the three $h / \delta_{o}$ ratios are presented in table 2 .

In figure 10 we present contours of turbulent kinetic energy are different stream-wise planes normal to the flow direction. An important point to remember while viewing figure 10 is that the viewing angle is adjusted so that all the three cases appear similar. In reality the physical dimensions of the three cases are different and scale with the cube height $h$. As we move along the stream-wise direction we observe two things happening: one, the maximum value of TKE decreases due to dissipation, and two, TKE spreads over a larger spanwise area due to convection. At a distance of $1 h$ behind the cube we observe a large magnitude of TKE. At a distance of $3 h$ behind the cube the magnitude of TKE for $h / \delta_{o}=1.0$ is slightly greater than the other 2 cases. Further downstream at a distance of $6 h$ from the cube we observe a more uniform distribution of TKE inside the boundary layer due to convection. Also, the magnitude is lower due to dissipation. From the contour plots in figure 10 and TKE density plot in figure 11 we realize that the magnitude of TKE for different $h / \delta_{o}$ ratios are comparable to each other, however the value of TKE is more concentrated in smaller region behind the cube for $h / \delta_{o}=0.6$ and 0.2 . The larger magnitude of TKE for $h / \delta=1.0$ gets distributed over a larger area making it least effective in TKE entrainment per unit area.

The presence of the cube induces vortices capable of entraining turbulent kinetic energy (TKE) from the mean flow to the near wall region. As such, the cube acts as a passive vortex generator (VG). In figure 11 we sample the TKE at different planes normal to the streamwise direction from the bottomwall to a height of $1.6 \delta_{o}$ and the entire span of the domain. We add all these values in the $y-z$ plane. The TKE values are normalized by the square of the mean velocity $U_{b}$ and divided by the area of the plane stretching over $7 h$ in span and $1.6 \delta_{o}$ in height. The area is normalized by the square of the boundary layer thickness at the cube, which is $\delta_{o}$. From figure 11 we observe that TKE per unit area is same for all three cases at a distance of

\begin{tabular}{llcc}
\hline \hline$h / \delta_{o}$ & $C_{d}$ & Separation length $\left(X_{F} / h\right)$ & Reattachment length $\left(X_{R} / h\right)$ \\
\hline 0.2 & 0.6 & 1.3 & 1.6 \\
0.6 & 0.9 & 1.2 & 1.8 \\
1 & 1.0 & 1.5 & 1.6 \\
\hline \hline
\end{tabular}

${ }^{*} C_{d}$ : Drag coefficient

Table 2. Separation and reattachment length normalized by cube height for a spatially evolving turbulent boundary layer flow over a wall-mounted cube. 


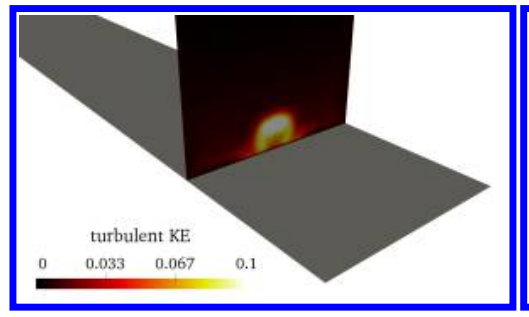

(a) $h / \delta_{o}=0.2, x=h$

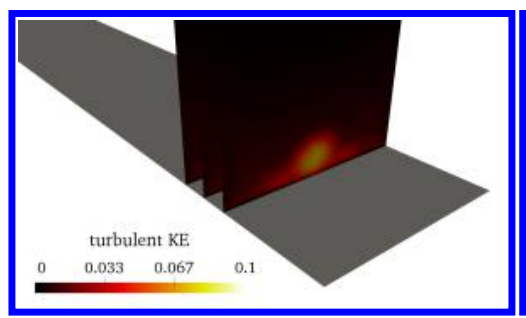

(d) $h / \delta_{o}=0.2, x=3 h$

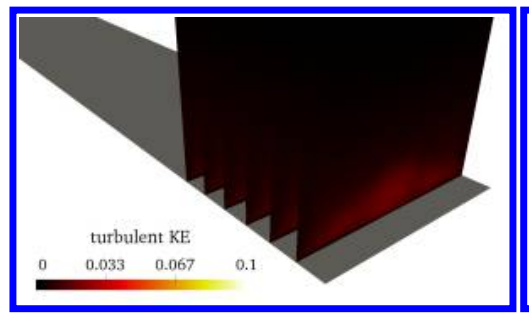

(g) $h / \delta_{o}=0.2, x=6 h$

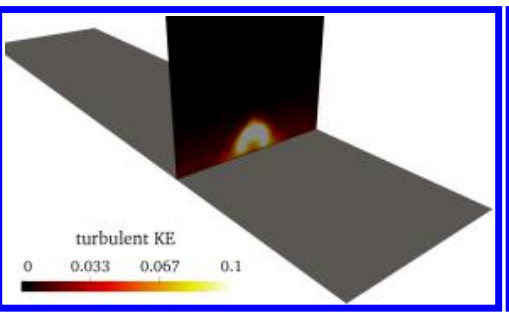

(b) $h / \delta_{o}=0.6, x=h$

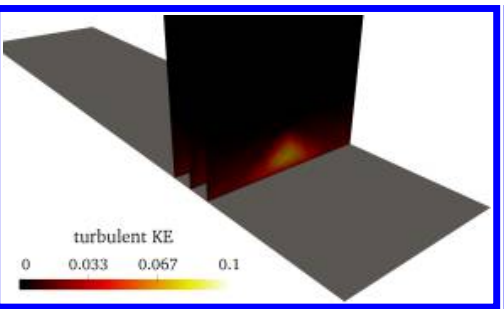

(e) $h / \delta_{o}=0.6, x=3 h$

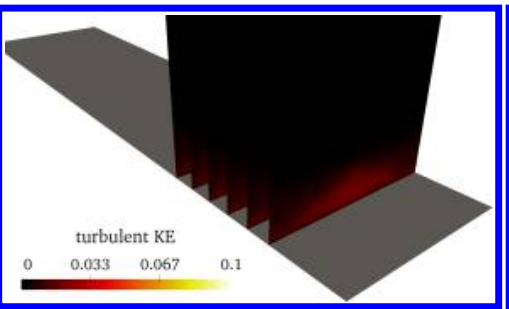

(h) $h / \delta_{o}=0.6, x=6 h$

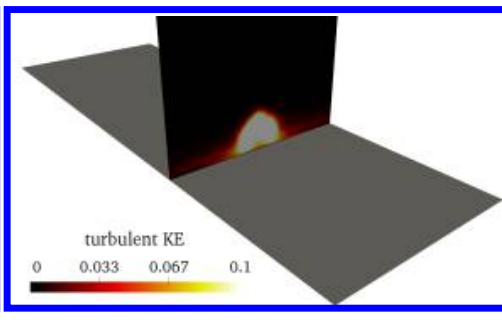

(c) $h / \delta_{o}=1.0, x=h$

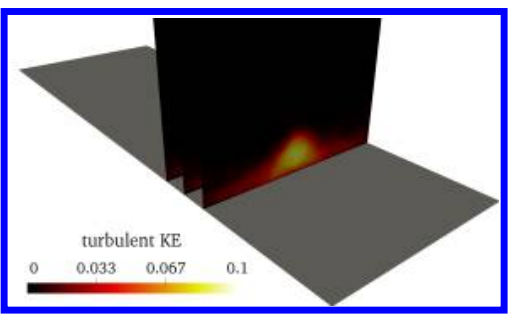

(f) $h / \delta_{o}=1.0, x=3 h$

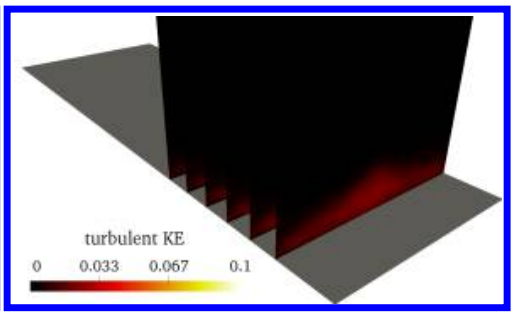

(i) $h / \delta_{o}=1.0, x=6 h$

Figure 10. Contours of turbulent kinetic energy at different stream-wise locations. (a), (d) and (g) are for $h / \delta_{o}=0.2$, (b), (e) and (h) are for $h / \delta_{o}=0.6$ and (c), (f) and (i) are for $h / \delta_{o}=1.0$. The figures in the top row are planes at a distance of $1 h$ from the cube, figures in the middle row are planes at a distance of $3 h$ from the cube and figures in the bottom row are planes at a distance of $6 h$ from the cube.

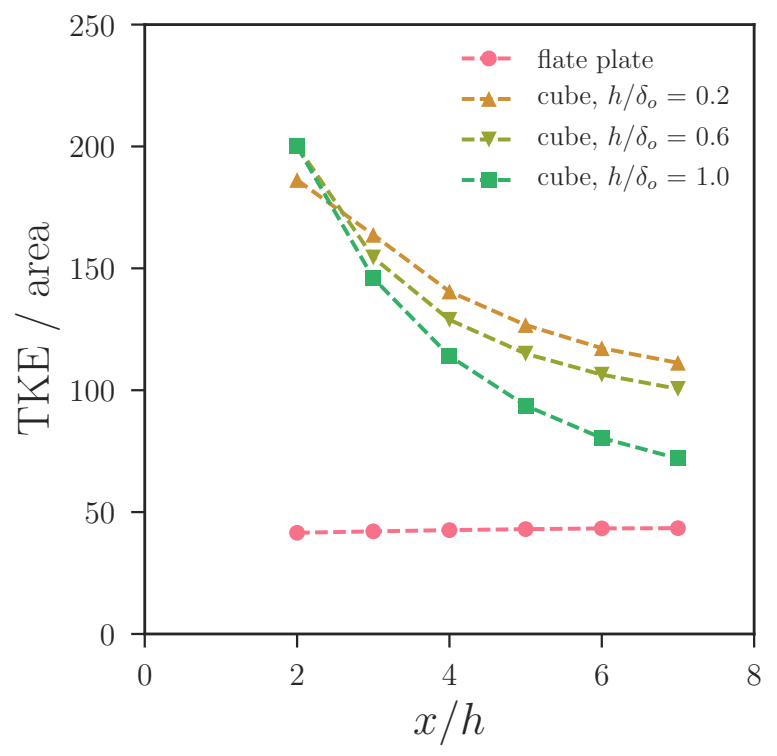

Figure 11. Average turbulent kinetic energy (TKE) per unit area as a function of the streamwise coordinate

$1 h$ from the back-face of the cube. As we move along the streamwise direction the TKE area density for $h / \delta=1.0$ decrease most rapidly followed by $h / \delta=0.6$ and finally 0.2 . This indicates that there exist higher 

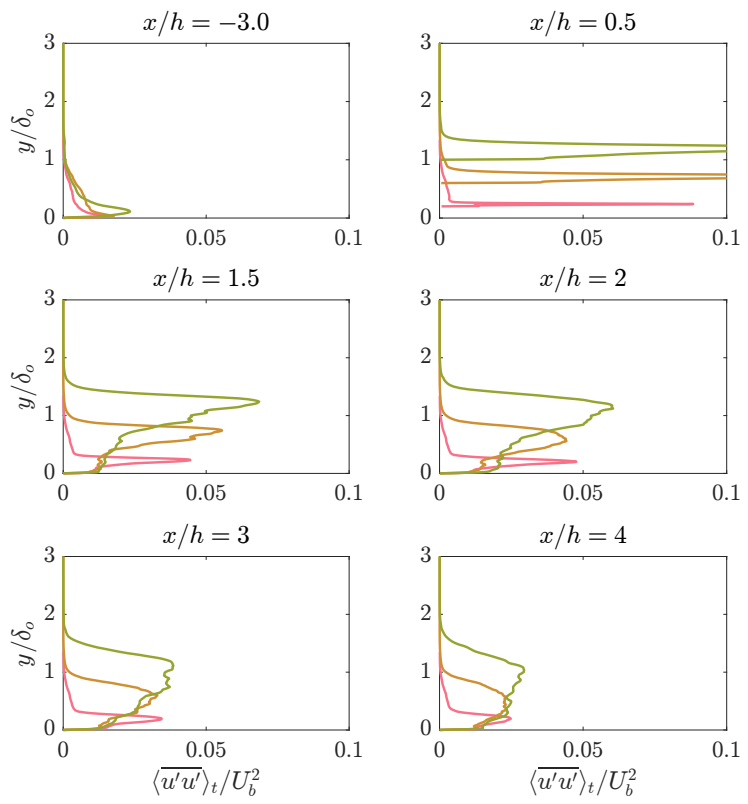

(a) $x x$-component of Reynolds stress
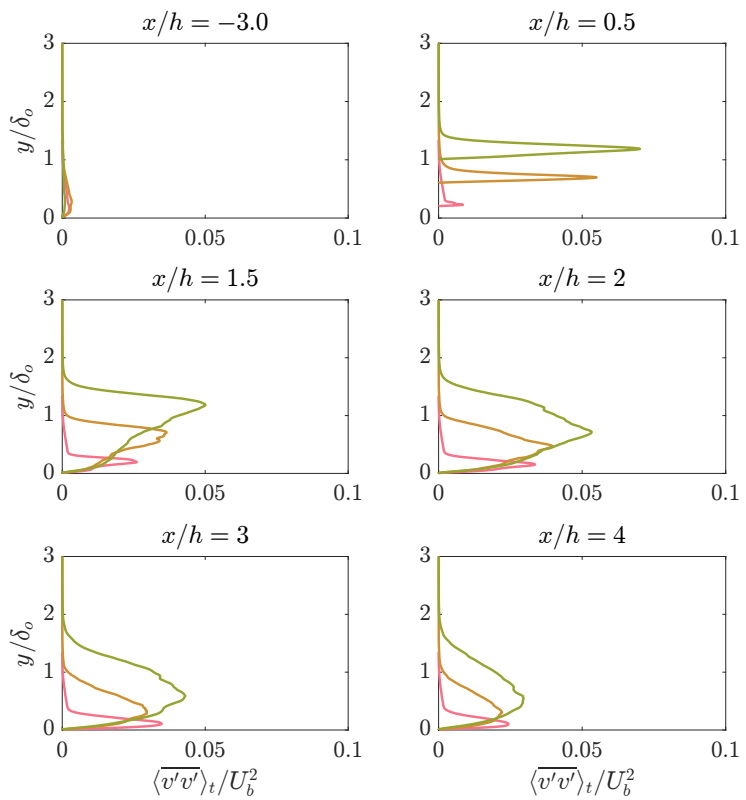

(b) yy-component of Reynolds stress

Figure 12. Turbulence statstics for a spatially evolving turbulent boundary layer flow around a wall-mounted cube. $h / \delta_{o}=0.2,-h / \delta_{o}=0.6,-h / \delta_{o}=1.0$.

TKE dissipation for a cube with larger height. From the statistical derivation of the $k$-equation LES model explained by Yoshizawa et al. ${ }^{18}$ we know that the $\mathrm{KE}$ dissipation rate $\epsilon \sim k^{3 / 2}$, where $k$ is the sub-grid scale (SGS) kinetic energy. The production of $k$ is directly proportional to the velocity gradient. ${ }^{18}$ This helps us to explain the pattern observed in figure 11. For a larger cube the shear layer formed on the top of the cube is surrounded by higher velocity fluid. Therefore, a larger cube has steeper velocity gradients on its top surface, giving rise to more production of SGS $k$ and subsequently more dissipation of TKE. Thus, a larger cube has smaller TKE area density.

In figure 12 we present the $x x$ - and $y y$-components of Reynolds stress at different stream-wise locations in the plane of symmetry for the 3 cube heights. Two main observations are that the peak value increases with increasing cube height and the position of the peak value also increases with increasing cube height. The magnitude of the peak value depends on the magnification of the disturbances introduced by the cube. Disturbances are proportional to the cube size. Thus, a larger cube introduces disturbances at greater distances from the bottomwall where the background velocity is higher leading to a greater correlation in the Reynolds stresses. Also, the position of the peak value depends on the position of the shear layer formed from the top surface of the cube. A close look at the magnitude of the $x x$-component near the wall reveals that the value remains almost constant. This is due to the fact that the rate of recovery is much faster in the inner region of the boundary layer. Similar observations were made by Wasistho et al. ${ }^{19}$ on a backward facing smooth ramp.

The $x x$-component of the Reynolds stress decreases for all three cube heights as we move along the stream-wise direction. This is expected because the turbulent kinetic energy gets dissipated as the boundary layer recovers. The same can be said about the $y y$-component. However, an interesting phenomenon is observed in the $y y$-component for the $h / \delta_{o}=0.2$ case. The magnitude of this component remains almost constant at all the stream-wise locations after the cube. It has been observed that the $y y$-component exhibits slower recovery as compared to the other Reynolds stress components in flow over a smooth ramp. ${ }^{19}$ It is speculated to be because of the farther distance of the peak for the $y y$-component away from the wall. It is unclear why this behavior is observed only for the $h / \delta_{o}=0.2$ case. 


\section{Conclusions and future work}

In this study we validated our flow solvers by comparing our results to simulations and experiments of a the wall-mounted cube in channel flow. The results are in good agreement with experimental and numerical results from prior studies. We also tested our inflow generation method by performing a highfidelity simulation of flow over a flat plate at $R e_{\tau}=800$. We are able to replicate a spatially evolving turbulent boundary layer with reasonable accuracy. The main focus of the present study is to understand the effect of varying the cube height to boundary layer thickness ratio $\left(h / \delta_{o}\right)$ on the flow characteristics at an inlet Reynolds number of 19,600. We present our results for three $h / \delta_{o}$ ratios namely, $0.2,0.6$ and 1.0 on three grids with increasing refinement levels.

Our results indicate that the separation and reattachment length of the flow is independent of the $h / \delta_{o}$ ratio. The flow reattaches at a distance of $\sim 1.8 h$ after the cube. The streamline plots reveal that the size of the arch-type vortex formed behind the cube decreases with increasing $h / \delta_{o}$ ratio. The horse-shoe vortex in front of the cube extends to a distance of $3 h$ around the cube. The drag coefficient $\left(C_{d}\right)$ acting on the cube increases with increasing cube height. The cube with $h=0.2 \delta_{o}$ entrains the most turbulent KE inside the boundary layer per unit area as compared to the other two cases due to higher dissipation of TKE in the shear layer on the top of a cube with large size. The $x x$ - and $y y$-components of Reynolds stresses in the center-plane show different behavior. The $x x$-component recovers at a faster rate closer to the wall as compared to the shear-layer, whereas the $y y$-component does not exhibit this fast recovery. The $y y$-component of Reynolds stress for the case with $h / \delta_{o}=0.2$ decays by negligible amount over a streamwise distance of $4 h$ as compared to the cases with $h / \delta_{o}=0.6$ and 1 . In the future we propose to understand effect of the distance between multiple cubes when placed next to each other on the flow characteristics.

\section{Acknowledgements}

The authors would like to thank Dr. Pooya Movahed for his contribution in developing the inflow conditions. This work used the Extreme Science and Engineering Discovery Environment (XSEDE), which is supported by National Science Foundation grant number ACI-1053575.

\section{Appendix: Grid refinement study}

To establish grid independence, we used three grids for each of the three different cube heights. The grids are labelled as coarse, medium and fine. The non-dimensional grid spacing is the same for the three different cube heights. The details of the grid spacing are summarized in table 3 .

In figures 13,14 and 15 we present the streamwise component of mean velocity and $x x$-component of Reynolds stress for three cube heights $h / \delta_{o}=0.2,0.6$ and 1.0 on the coarse, medium and fine mesh respectively to determine how well resolved the simulations are. For $h / \delta_{o}=0.2$, we observe greater deviation in the $x$-component of mean velocity in the coarse mesh from the medium and fine mesh than for the larger values of $h / \delta_{o}$. The reason for this greater difference in the $h / \delta_{o}=0.2$ case is the same non-dimensional grid spacing used across all the three cases, which results in fewer cells across the cube height in the coarse mesh for the smallest $h / \delta_{o}$. The results for $x$-component of mean velocity from medium and fine mesh cases are in good agreement for all the 3 cube heights.

We observe noticeable deviations in the $x x$-component of Reynolds stress between the coarse, medium and fine mesh cases in figures 13(b), 14(b) and 15(b). Since Reynolds stresses are calculated as the mean of

\begin{tabular}{lccc}
\hline \hline Property & coarse mesh & medium mesh & fine mesh \\
\hline$\Delta_{x}^{+}$ & 22 & 18 & 15 \\
$\Delta_{z}^{+}$ & 22 & 18 & 15 \\
$\Delta_{y}^{+}$ & 5 & 1 & 1 \\
\hline \hline
\end{tabular}

Table 3. Non-dimensional grid spacing details 

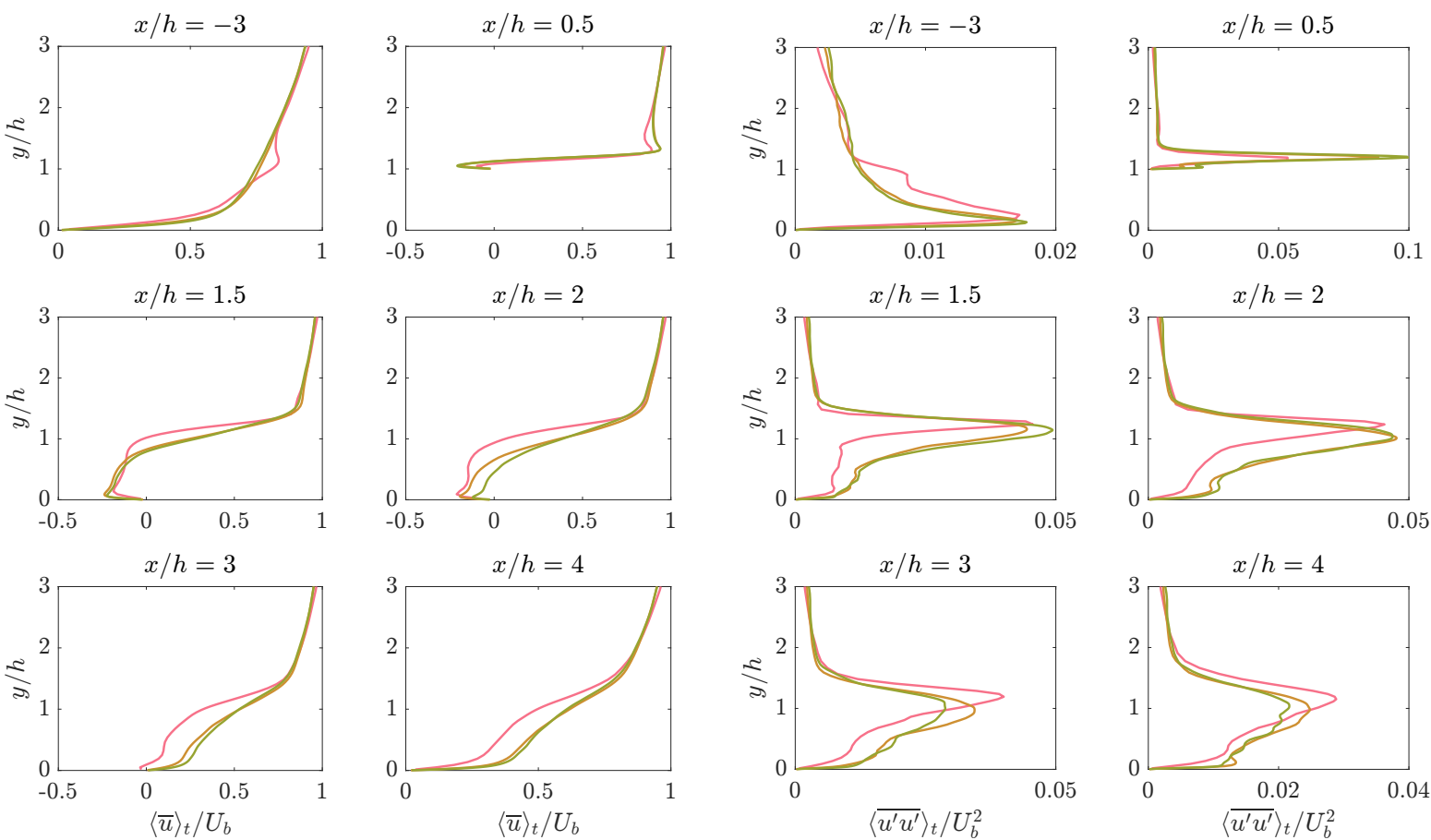

(a) $x$-component of mean velocity
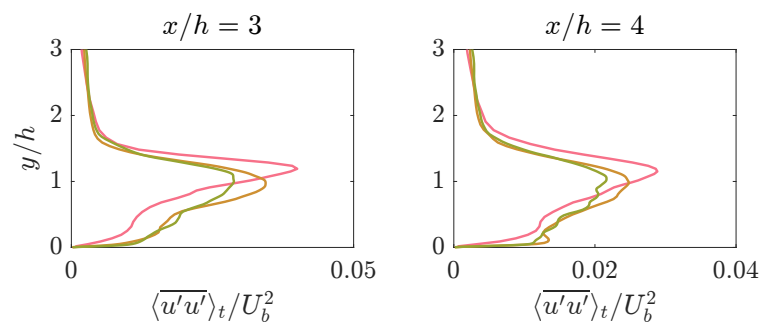

(b) $x x$-component of Reynolds stress

Figure 13. Profiles of mean velocity ( $x$-component) and Reynolds stress ( $x x$-component) for spatially evolving turbulent boundary layer flow around a wall-mounted cube with $h / \delta_{o}=0.2$ - coarse mesh, — medium mesh, — fine mesh
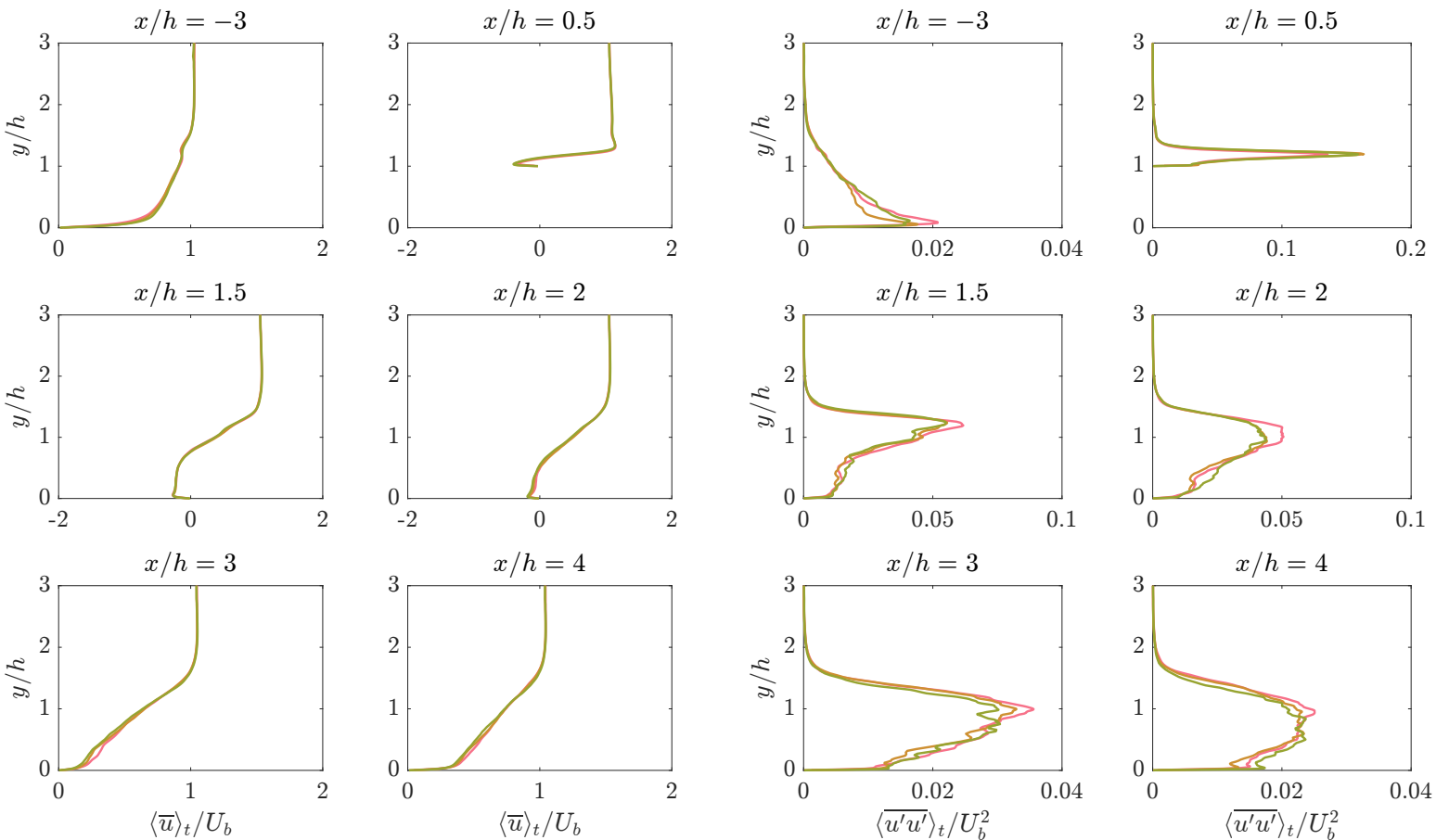

(a) $x$-component of mean velocity
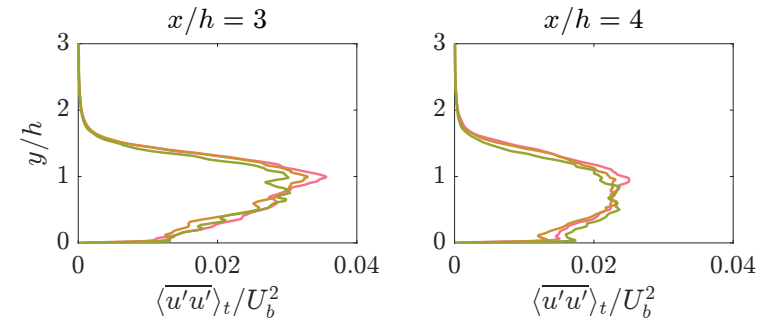

(b) $x x$-component of Reynolds stress

Figure 14. Profiles of mean velocity ( $x$-component) and Reynolds stress ( $x x$-component) for spatially evolving turbulent boundary layer flow around a wall-mounted cube with $h / \delta_{o}=0.6$.

coarse mesh,

medium mesh, mesh 

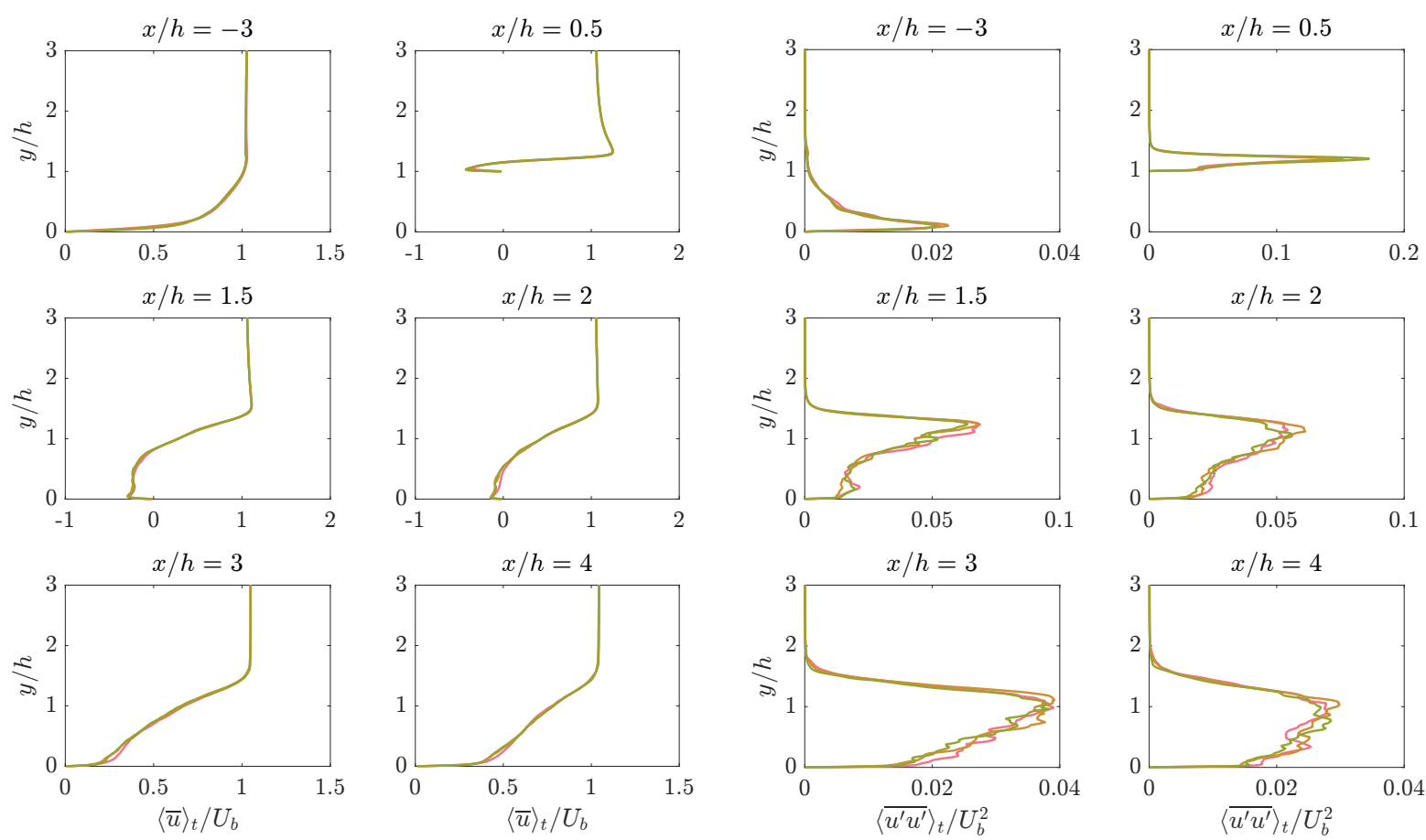

(a) $x$-component of mean velocity
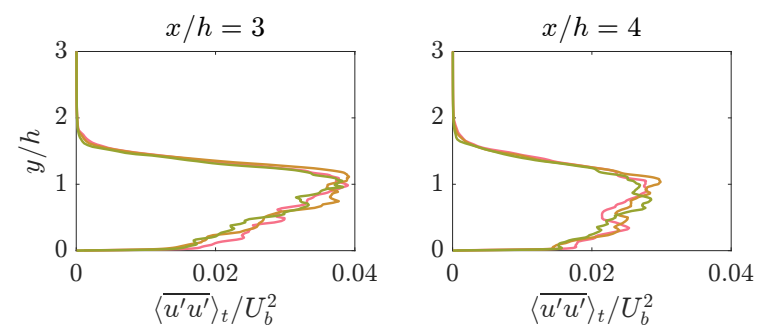

(b) $x x$-component of Reynolds stress

Figure 15. Profiles of mean velocity ( $x$-component) and Reynolds stress ( $x x$-component) for spatially evolving turbulent boundary layer flow around a wall-mounted cube with $h / \delta_{o}=1.0$. - coarse mesh, — medium mesh, — fine mesh

the difference between instantaneous and time-average velocities, the magnitude of values under consideration are small. As a result any slight deviations get amplified. According to our experience and from the literature, we believe that the results in figures 13(b), 14(b) and 15(b) are acceptable and that the finest mesh accurately represents the flow dynamics.

\section{References}

${ }^{1}$ Krajnovic, S. and Davidson, L., "Large-eddy simulation of the flow around a bluff body," AIAA journal, Vol. 40, No. 5, 2002, pp. 927-936.

${ }^{2}$ Shah, K. B., Large eddy simulations of flow past a cubic obstacle, Ph.D. thesis, Stanford University, 1998.

${ }^{3}$ Krajnovic, S. and Davidson, L., "Large Eddy Simulation of Flow Around a Surface-Mounted Cube using a Dynamic One-Equation Subgrid Model," Journal of Chemical Information and Modeling, 1999.

${ }^{4}$ Rodi, W., "Comparison of LES and RANS calculations of the flow around bluff bodies," Journal of Wind Engineering and Industrial Aerodynamics, Vol. 69-71, 1997, pp. 55-75.

${ }^{5}$ Iaccarino, G., Ooi, A., Durbin, P., and Behnia, M., "Reynolds averaged simulation of unsteady separated flow," International Journal of Heat and Fluid Flow, Vol. 24, No. 2, 2003, pp. 147-156.

${ }^{6}$ Yazid, A. W. M. and Sidik, N. A. C., "Prediction of the Flow around a Surface-Mounted Cube Using Two-Equation Turbulence Models," Applied Mechanics and Materials, Vol. 315, 2013, pp. 438-442.

${ }^{7}$ Martinuzzi, R. and Tropea, C., "The flow around surface-mounted, prismatic obstacles placed in a fully developed channel flow," Journal of Fluids Engineering, Vol. 115, No. 1, 1993, pp. 85-92.

${ }^{8}$ Hussein, H. J. and Martinuzzi, R., "Energy balance for turbulent flow around a surface mounted cube placed in a channel," Physics of Fluids, Vol. 8, No. 3, 1996, pp. 764-780.

${ }^{9}$ Shah, K. B. and Ferziger, J. H., "A fluid mechanicians view of wind engineering: Large eddy simulation of flow past a cubic obstacle," Journal of Wind Engineering and Industrial Aerodynamics, Vol. 67, 1997, pp. 211-224.

${ }^{10}$ Liakos, A. and Malamataris, N. A., "Direct numerical simulation of steady state, three dimensional, laminar flow around a wall mounted cube," Physics of Fluids, Vol. 26, No. 5, 2014, pp. 053603.

${ }^{11}$ Yakhot, A., Liu, H., and Nikitin, N., "Turbulent flow around a wall-mounted cube: A direct numerical simulation," International Journal of Heat and Fluid Flow, Vol. 27, No. 6, dec 2006, pp. 994-1009.

12 Yakhot, A., Anor, T., Liu, H., and Nikitin, N., "Direct numerical simulation of turbulent flow around a wall-mounted cube: spatio-temporal evolution of large-scale vortices," Journal of Fluid Mechanics, Vol. 566, No. 1, 2006, pp. 1-9.

${ }^{13}$ Lim, H. C., Thomas, T. G., and Castro, I. P., "Flow Around a Cube in a Turbulent Boundary Layer LES and Experiment," Journal of Wind Engineering and Industrial Aerodynamics, , No. January 2008, 2009, pp. 625-628. 
${ }^{14} \mathrm{Wu}, \mathrm{X}$. and Moin, P., "Direct numerical simulation of turbulence in a nominally zero-pressure-gradient flat-plate boundary layer," Journal of Fluid Mechanics, Vol. 630, 2009, pp. 5-41.

${ }^{15}$ Shinde, S., Tandon, S., Maki, K., and Johnsen, E., "Flow separation over a backward-facing ramp with and without a vortex generator," 46th AIAA Fluid Dynamics Conference, AIAA Aviation, American Institute of Aeronautics and Astronautics, June 2016.

${ }^{16}$ Ferrante, A. and Elghobashi, S., "A robust method for generating inflow conditions for direct simulations of spatiallydeveloping turbulent boundary layers," Journal of Computational Physics, Vol. 198, No. 1, 2004, pp. $372-387$.

${ }^{17}$ De Graaff, D. B. and Eaton, J. K., "Reynolds-number scaling of the flat-plate turbulent boundary layer," Journal of Fluid Mechanics, Vol. 422, 2000, pp. 319-346.

${ }^{18}$ Yoshizawa, A. and Horiuti, K., "A statistically-derived subgrid-scale kinetic energy model for the large-eddy simulation of turbulent flows," Journal of the Physical Society of Japan, Vol. 54, No. 8, 1985, pp. 2834-2839.

${ }^{19}$ Wasistho, B. and Squires, K., "Prediction of turbulent separation over a backward-facing smooth ramp," Journal of Turbulence, , No. 6, 2005, pp. N1. 